Ferrata Storti Foundation

\title{
First-line therapy with either bortezomib- melphalan-prednisone or lenalidomide- dexamethasone followed by lenalidomide for transplant-ineligible multiple myeloma patients: a pooled analysis of two randomized trials
}

Haematologica 2020

Volume 105(4):1074-1080

\section{Correspondence: \\ ALESSANDRA LAROCCA \\ alelarocca@hotmail.com \\ Received: February 27, 2019. \\ Accepted: June 25, 2019 \\ Pre-published: June 27, 2019.}

doi:10.3324/haematol.2019.220657

Check the online version for the most updated information on this article, online supplements, and information on authorship \& disclosures: www. haematologica.org/content/105/4/1074

(C)2020 Ferrata Storti Foundation

Material published in Haematologica is covered by copyright. All rights are reserved to the Ferrata Storti Foundation. Use of published material is allowed under the following terms and conditions:

https://creativecommons.org/licenses/by-nc/4.0/legalcode. Copies of published material are allowed for personal or internal use. Sharing published material for non-commercial purposes is subject to the following conditions:

https://creativecommons.org/licenses/by-nc/4.0/legalcode, sect. 3. Reproducing and sharing published material for commercial purposes is not allowed without permission in writing from the publisher.
Alessandra Larocca, ${ }^{1}$ Roberto Mina, ${ }^{1}$ Massimo Offidani, ${ }^{2}$ Anna Marina Liberati, ${ }^{3}$ Antonio Ledda, ${ }^{4}$ Francesca Patriarca, ${ }^{5}$ Andrea Evangelista, ${ }^{6}$ Stefano Spada, ${ }^{1}$ Giulia Benevolo, ${ }^{7}$ Daniela Oddolo, ${ }^{1}$ Vanessa Innao, ${ }^{8}$ Clotilde Cangiolosi, ${ }^{9}$ Annalisa Bernardini, ${ }^{1}$ Pellegrino Musto, ${ }^{10}$ Valeria Amico, ${ }^{11}$ Vincenzo Fraticelli, ${ }^{12}$ Laura Paris, ${ }^{13}$ Nicola Giuliani, ${ }^{14}$ Antonietta Pia Falcone, ${ }^{15}$ Renato Zambello, ${ }^{16}$ Lorenzo De Paoli, ${ }^{17}$ Alessandra Romano, ${ }^{18}$ Antonio Palumbo, ${ }^{1 *}$ Vittorio Montefusco, ${ }^{19}$ Roman Hájek, ${ }^{20,21}$ Mario Boccadoro ${ }^{1}$ and Sara Bringhen ${ }^{1}$

${ }^{1}$ Myeloma Unit, Division of Hematology, University of Torino, Azienda-Ospedaliero Universitaria (AOU) Città della Salute e della Scienza di Torino, Torino, Italy; ${ }^{2}$ Clinica di Ematologia, AOU Ospedali Riuniti di Ancona, Ancona, Italy; ${ }^{3}$ Università degli Studi di Perugia, Azienda Ospedaliera (AO) Santa Maria, Terni, Italy; ${ }^{4}$ Ematologia/CTMO Ospedale "A. Businco", Cagliari, Italy; ${ }^{5}$ Udine University Hospital, DAME, University of Udine, Udine, Italy; ${ }^{6}$ Unit of Clinical Epidemiology, AOU Città della Salute e della Scienza di Torino e CPO, Piemonte, Torino, Italy; ${ }^{7}$ Hematology, Città della Salute e della Scienza, Torino, Italy; ${ }^{8}$ U.O.C. Ematologia AOU G. Martino, Messina, Italy; ${ }^{9} \mathrm{AO}$ Villa Sofia-Cervello, Divisione Ematologia I UTMO, Palermo, Italy; ${ }^{10}$ Unit of Haematology and Stem Cell Transplantation, IRCCS-CROB, Referral Cancer Center of Basilicata, Rionero in Vulture, Italy; ${ }^{11} \mathrm{DH}$-Ematologico AO "S.Pio", Benevento, Italy; ${ }^{12}$ Fondazione "Giovanni Paolo II", Unità Operativa Complessa di Oncoematologia, Campobasso, Italy; ${ }^{13} \mathrm{Hematology}$ and Bone Marrow Transplant Unit, Azienda Socio Sanitaria Territoriale Papa Giovanni XXIII, Bergamo, Italy; ${ }^{14}$ Department of Medicine and Surgery, University of Parma, Parma, Italy; ${ }^{15}$ Ematologia, IRCCS "Casa Sollievo della Sofferenza" Hospita, San Giovanni Rotondo, Italy; ${ }^{16}$ Padova University School of Medicine, Hematology and Clinical Immunology, Padova, Italy; ${ }^{17}$ Università del Piemonte Orientale A. Avogadro, Novara, Italy; ${ }^{18}$ Division of Hematology, AOU Policlinico, Department of Surgery and Medical Specialties, University of Catania, Italy; ${ }^{19} \mathrm{Hematology,} \mathrm{Fondazione} \mathrm{IRCCS} \mathrm{Istituto}$ Nazionale Tumori, Milan, Italy; ${ }^{20}$ Department of Hematooncology University Hospital Ostrava, Ostrava, Czech Republic and ${ }^{21}$ Faculty of Medicine, University of Ostrava, Ostrava, Czech Republic

${ }^{\star}$ Currently a GlaxoSmithKline AG employee.

\section{ABSTRACT}

B ortezomib-melphalan-prednisone (VMP) and continuous lenalidomide-dexamethasone $(\mathrm{Rd})$ represent the standard treatment of transplant-ineligible patients with newly diagnosed multiple myeloma (MM). To date, no randomized trial has compared VMP to Rd, and there is no evidence of the optimal treatment for newly diagnosed $\mathrm{MM}$, particularly in patients with high-risk cytogenetics [del(17p), $\mathrm{t}(4 ; 14)$ or $t(14 ; 16)]$. We pooled together data from patients with newly diagnosed MM treated with VMP or Rd induction followed by lenalidomide maintenance $10 \mathrm{mg}(\mathrm{Rd}-\mathrm{R})$ enrolled in the GIMEMA-MM-03-05 and EMN01 trials, to evaluate the efficacy of these treatments in different subgroups of patients, focusing on those with standard- and high-risk cytogenetics. Overall, 474 patients were analyzed (VMP: 257 patients; Rd-R: 217 patients). No differences in progression-free survival (hazard ratio $=0.96$ ) and overall survival (hazard ratio=1.08) were observed between standard-risk patients treated with VMP or Rd-R, whereas among the high-risk patients, the probabilities of progression (hazard ratio $=0.54$ ) and death (hazard ratio $=0.73$ ) were lower in the patients treated with VMP than in those treated with $\mathrm{Rd}-\mathrm{R}$. In particular, standard-risk patients $>75$ years benefited less from VMP than from Rd-R 
(hazard ratio for progression-free survival=0.96; hazard ratio for overall survival=1.81). In this non-randomized analysis, VMP and Rd-R were equally effective in younger ( $\leq 75$ years), standard-risk patients, while older ones (>75 years) benefited more from Rd-R. In high-risk patients, VMP improved progression-free survival and overall survival irrespective of age. The source trials are registered at ClinicalTrials.gov (NCT01063179 and NCT01093196).

\section{Introduction}

Multiple myeloma ( $\mathrm{MM})$ is a disease that occurs predominantly in the elderly: the median age at diagnosis is 71 years and two-thirds of patients are over 65 years of age. ${ }^{1,2}$ Elderly patients, defined as those older than 65-70 years of age, are usually considered ineligible for highdose chemotherapy and autologous stem-cell transplantation (ASCT). ${ }^{3}$ In Europe, standard initial therapy of older patients consists of either a triplet regimen including bortezomib-melphalan-prednisone (VMP) administered in a fixed-duration schedule, or a doublet combination of lenalidomide $(25 \mathrm{mg}$ ) and dexamethasone (Rd), administered continuously until progression or intolerance. ${ }^{3}$ The VISTA trial demonstrated that VMP was superior to melphalan-prednisone both in terms of progression-free survival [PFS: 21.7 vs. 15.2 months, hazard ratio $(\mathrm{HR})=0.56$; $P<0.001]$ and overall survival (OS: 56.4 vs. 43.1 months, $\mathrm{HR}=0.69 ; P<0.001){ }^{4,5}$ The FIRST trial showed that continuous Rd significantly prolonged the median PFS (26 vs. 21.9 months, $\mathrm{HR}=0.69 ; P<0.001)$ and $\mathrm{OS}(59.1$ vs. 49.1 months, HR=0.78; $P=0.0023$ ) compared to the median PFS and $O S$ achieved with melphalan-prednisone-thalidomide (MPT) ${ }^{6,7}$ Based on the results of these two phase III studies, both VMP and continuous Rd were approved by the European Medicine Agency as standard treatments for patients with newly diagnosed MM ineligible for ASCT.

The most important prognostic factors in $\mathrm{MM}$ are age and frailty, disease stage defined by the International Staging System (ISS), ${ }^{8}$ and chromosomal abnormalities, detected by fluorescent in situ hybridization (FISH). Patients harboring chromosomal abnormalities, including del(17p), $t(4,14)$ and $t(14,16)$, have a poor prognosis, with a higher risk of disease progression and death. ${ }^{9}$ Approximately $15-20 \%$ of patients with newly diagnosed MM present with at least one cytogenetic abnormality and constitute the so-called "high-risk" population.

Despite recent therapeutic advances in $M M$, the results obtained with novel agent-based regimens in patients with high-risk chromosomal abnormalities are unsatisfactory and the prognosis of these patients remains poor. Moreover, limited data are available on high-risk, transplant-ineligible MM patients treated with bortezomib or lenalidomide in first-line therapy. In the VISTA trial, patients with high- or standard-risk cytogenetics who received VMP had similar times to progression (median 19.8 vs. 23.1 months, $\mathrm{HR}=1.29 ; P=0.55)$ and $\mathrm{OS}(\mathrm{HR}=1.00$; $P=0.99) .{ }^{4,5}$ In a subanalysis of the FIRST trial, which compared continuous $\mathrm{Rd}$ treatment until progression, $\mathrm{Rd}$ treatment for 72 weeks (Rd18) and MPT treatment, continuous $\mathrm{Rd}$ treatment resulted in PFS and OS benefits in comparison with MPT; however, these benefits were largely due to the improvements in PFS and OS in patients without high-risk cytogenetics (median PFS 31.1 vs. 21.2 vs. 24.9 months for continuous Rd vs. Rd18 vs. MPT). Indeed, in the high-risk group, the longest PFS was observed with
Rd18 treatment (median 8.4 vs. 17.5 vs. 14.6 months for continuous $\mathrm{Rd}$ vs. Rd18 vs. MPT) while OS was similar across treatment arms. ${ }^{10}$

Unfortunately, VMP and Rd have never been formally compared in a randomized trial. Based on the different safety profiles of bortezomib and lenalidomide, bortezomib is usually preferred in patients with advanced renal failure, while lenalidomide can be the drug of choice for patients with pre-existing neuropathy, or when oral therapy is preferable. ${ }^{11}$ Besides these considerations, VMP and Rd are equally recommended. Because a head-to-head comparison of VMP vs. Rd is lacking, the choice of firstline treatment of elderly MM patients is based mainly on the physician's and patient's preferences.

We previously published the results of two randomized, phase III studies investigating both VMP (GIMEMA-MM03-05) $)^{12-14}$ and Rd induction followed by lenalidomide maintenance (Rd-R) (EMN01) ${ }^{15}$ as upfront therapies for elderly, transplant-ineligible MM patients. In order to provide clinicians with useful, and currently lacking, evidence that may help to tailor anti-myeloma treatment better in this population, we conducted a pooled, retrospective analysis comparing the efficacy of VMP and Rd-R in different subgroups of elderly, transplant-ineligible MM patients, focusing on cytogenetic profile.

\section{Methods}

\section{Study design and participants}

We pooled the single data from two phase III studies, the GIMEMA-MM-03-05 (NCT01063179) and the EMN01 (NCT01093196) trials. Both trials enrolled patients with newly diagnosed MM who were older than 65 years of age or younger but ineligible for ASCT. Inclusion and exclusion criteria, as well as treatment details of the source studies, have been published previously. ${ }^{12-15}$ Further details on study treatments and procedures are reported in the Online Supplementary Appendix. For this retrospective, not pre-planned analysis, we selected only patients randomized to VMP or Rd-R. The source studies were approved by the institutional review boards at each of the participating centers. All patients gave written informed consent before entering the source studies, which were performed in accordance with the Declaration of Helsinki.

\section{Statistical analysis}

The primary objective of this analysis was to compare PFS and OS (see Online Supplementary Appendix) in patients treated with VMP or Rd-R, adjusting for patient and disease characteristics at baseline.

Single data from the two studies were pooled together and analyzed. Comparisons between different groups of patients were conducted using standard statistical tests. Time-to-event data were analyzed using the Kaplan-Meier method; survival curves were compared with the log-rank test. Results are presented as hazard ratios (HR), 95\% confidence intervals (95\% CI), and two- 
sided $P$-values adjusted for age, ISS stage, cytogenetic risk as determined by FISH, Karnofsky Performance Status (PS) and extramedullary disease (yes vs. no) (main model). Subgroup analyses were performed to determine the consistency of treatment effects of VMP vs. Rd-R in the main model in the different subgroups using interaction terms between treatment and cytogenetics [also with single deletion and translocation, del(17p), $\mathrm{t}(4 ; 14)$ and $\mathrm{t}(14 ; 16)]$, ISS stage (I vs. II/III), age ( $\leq 75$ vs. $>75$ years), Karnofsky PS (90-100 vs. 70-80 vs. 60) and extramedullary disease. The null hypothesis examined with the interaction test was that the HR of the comparison VMP vs. Rd-R would be the same in each subgroup. The models were adjusted for age as a continuous variable. Multivariate Cox models with a three-way interaction between treatment (VMP vs. Rd-R), cytogenetics (high-risk vs. standard-risk, missing vs. standard-risk) and age ( $\leq 75$ vs. $>75$ years) were performed to evaluate the effect of treatment in different cytogenetic and age subgroups. The models were adjusted for other factors included in previous analyses. The different effect of VMP vs. Rd-R in cytogenetic subgroups was confirmed by a sen-

Table 1. Baseline patients' characteristics in the intention-to-treat population.

\begin{tabular}{|c|c|c|c|c|}
\hline & $\begin{array}{c}\text { All patients } \\
(n=474)\end{array}$ & $\begin{array}{c}\text { VMP } \\
(n=257)\end{array}$ & $\begin{array}{c}\mathrm{Rd}-\mathrm{R} \\
(n=217)\end{array}$ & P-value \\
\hline $\begin{array}{c}\text { Age - median (IQR) } \\
>75 \text { years }-\mathrm{n}(\%)\end{array}$ & $\begin{array}{c}72(69-76) \\
131(28)\end{array}$ & $\begin{array}{l}71(68-75) \\
57(22)\end{array}$ & $\begin{array}{c}73(70-77) \\
74(34)\end{array}$ & $<0.001$ \\
\hline $\begin{array}{l}\text { Karnofsky PS - n (\%) } \\
90-100 \\
70-80 \\
60\end{array}$ & $\begin{array}{c}232(49) \\
200(42) \\
42(9)\end{array}$ & $\begin{array}{c}121(47) \\
118(46) \\
18(7)\end{array}$ & $\begin{array}{l}111(51) \\
82(38) \\
24(11)\end{array}$ & 0.11 \\
\hline Creatinine - mg/dL (IQR) & $1(0.8-1.22)$ & $1.01(0.84-1.3)$ & $0.94(0.8-1.19)$ & 0.002 \\
\hline $\begin{array}{c}\mathrm{LDH}-\mathrm{UI} / \mathrm{L}(\mathrm{IQR}) \\
\text { missing - n (\%) }\end{array}$ & $\begin{array}{l}286(202-377) \\
80(17)\end{array}$ & $\begin{array}{l}293(203-368) \\
\quad 36(14)\end{array}$ & $\begin{array}{c}284(200-381) \\
44(20)\end{array}$ & 0.753 \\
\hline $\begin{array}{l}\text { Extramedullary } \\
\text { disease - n (\%) }\end{array}$ & $58(12)$ & $37(14)$ & $21(10)$ & 0.124 \\
\hline $\begin{array}{l}\text { ISS - n (\%) } \\
\text { I } \\
\text { II } \\
\text { III } \\
\text { Missing }\end{array}$ & $\begin{array}{l}117(25) \\
184(39) \\
117(25) \\
56(12)\end{array}$ & $\begin{array}{l}56(22) \\
88(34) \\
57(22) \\
56(22)\end{array}$ & $\begin{array}{l}61(28) \\
97(45) \\
59(27)\end{array}$ & 0.96 \\
\hline $\begin{array}{l}\text { Cytogenetics - n (\%) } \\
\text { standard-risk } \\
\text { high-risk* } \\
\text { missing }\end{array}$ & $\begin{array}{l}273(58) \\
95(20) \\
106(22)\end{array}$ & $\begin{array}{l}136(53) \\
48(19) \\
73(28)\end{array}$ & $\begin{array}{l}137(63) \\
47(22) \\
33(15)\end{array}$ & 1 \\
\hline $\begin{array}{l}\operatorname{Del}(17 p)-n(\%) \\
\text { no } \\
\text { yes } \\
\text { missing }\end{array}$ & $\begin{array}{l}321(68) \\
47(10) \\
106(22)\end{array}$ & $\begin{array}{l}161(63) \\
23(9) \\
73(28)\end{array}$ & $\begin{array}{l}160(74) \\
24(11) \\
33(15)\end{array}$ & 1 \\
\hline $\begin{array}{l}\mathrm{t}(4 ; 14)-\mathrm{n}(\%) \\
\text { no } \\
\text { yes } \\
\text { missing }\end{array}$ & $\begin{array}{l}318(67) \\
50(11) \\
106(22)\end{array}$ & $\begin{array}{l}158(61) \\
26(10) \\
73(28)\end{array}$ & $\begin{array}{l}160(74) \\
24(11) \\
33(15)\end{array}$ & 0.88 \\
\hline $\begin{array}{l}\mathrm{t}(14 ; 16)-\mathrm{n}(\%) \\
\text { no } \\
\text { yes } \\
\text { missing }\end{array}$ & $\begin{array}{c}352(74) \\
13(3) \\
109(23)\end{array}$ & $\begin{array}{c}178(69) \\
6(2) \\
73(28)\end{array}$ & $\begin{array}{c}174(80) \\
7(3) \\
36(17)\end{array}$ & 0.785 \\
\hline $\begin{array}{l}\mathrm{t}(11 ; 14)-\mathrm{n}(\%) \\
\text { no } \\
\text { yes } \\
\text { missing }\end{array}$ & $\begin{array}{l}308(65) \\
54(11) \\
112(24)\end{array}$ & $\begin{array}{l}164(64) \\
20(8) \\
73(28)\end{array}$ & $\begin{array}{l}144(66) \\
34(16) \\
39(18)\end{array}$ & 0.038 \\
\hline
\end{tabular}

VMP: bortezomib-melphalan-prednisone; Rd-R: lenalidomide-dexamethasone followed by lenalidomide maintenance; IQR: interquartile range; PS: Performance Status; LDH: lactate dehydrogenase; ISS: International Staging System. *High-risk defined by the presence of any of $\operatorname{del}(17 \mathrm{p}), \mathrm{t}(4 ; 14)$, or $\mathrm{t}(14 ; 16)$, detected by fluorescence in situ hybridization. sitivity analysis using the multiple imputation method for missing cytogenetic and ISS values. In detail, missing data were handled using the "jomo" package ${ }^{16}$ to perform Cox model-compatible multiple imputation ${ }^{17,18}$ with 50 imputations, 1,000 burn-in iterations and 1,000 iterations between two successive imputations.

In both trials, FISH was assessed centrally with a $10 \%$ cutoff for numerical aberrations and a $15 \%$ one for IgH translocations. Highrisk cytogenetics was defined as the presence of at least one of the following chromosomal abnormalities: $\operatorname{del}(17 \mathrm{p}), \mathrm{t}(4 ; 14)$ or $\mathrm{t}(14 ; 16)$. Patients not carrying any of these abnormalities were defined as standard-risk patients. Data were censored on June 18, 2014 for the GIMEMA-MM-03-05 study and on October 20, 2017 for the EMN01 study. Data were analyzed using $\mathrm{R}$ software (version 3.5.1).

\section{Results}

\section{Patients}

A total of 474 patients were analyzed, 257 in the VMP group and 217 in the Rd-R group. Patients' demographic and baseline characteristics were well balanced between the two groups (Table 1 ). The median age of the overall population was 72 years [interquartile range (IOR) 69-76), with patients in the Rd-R group being slightly older (median, 73 years; IOR 70-77) than patients in the VMP group (median, 71 years; IOR 68-75; $P<0.001$ ). Similar proportions of patients in the VMP and $\mathrm{Rd}-\mathrm{R}$ groups had ISS stage III disease $(23 \%$ vs. $27 \%$; $P=0.74)$ and high-risk cytogenetics as determined by FISH ( $19 \%$ vs. $22 \%$; $P=1)$.

All patients started the assigned treatment. Overall, $61 \%$ and $64 \%$ of patients in the VMP and Rd-R groups, respectively, received a second-line treatment, which consisted of an immunomodulatory drug (IMiD)-based regimen in $63 \%$ of patients treated with VMP and of a proteasome inhibitor (PI)-based regimen in $84 \%$ of patients treated with $\mathrm{Rd}-\mathrm{R}$ (Table 2).

The median follow-up for the entire study population was 70.2 months (IOR 54.7-80.6), without a significant difference between the VMP and $\mathrm{Rd}-\mathrm{R}$ groups (72.6 vs. 64.4 months, respectively; $P=0.16$ ).

\section{Survival outcomes}

The median PFS in the overall population was 21.5 months (95\% CI: 19.8-24.9) and was not significantly different between patients treated with VMP (25.1 months,

Table 2. Second-line treatment.

\begin{tabular}{|c|c|c|c|c|}
\hline & $\begin{array}{c}\text { All patients } \\
(n=474)\end{array}$ & $\begin{array}{c}\text { VMP } \\
(n=257)\end{array}$ & $\begin{array}{c}R d-R \\
(n=217)\end{array}$ & P-value \\
\hline Second-line treatment $-\mathrm{n}(\%)$ & & & & 0.70 \\
\hline Yes & $296(62)$ & $158(61)$ & $138(64)$ & \\
\hline No & $178(38)$ & $99(39)$ & $79(36)$ & \\
\hline \multicolumn{5}{|l|}{ Type of treatment $-\mathrm{n}(\%)$} \\
\hline \multicolumn{5}{|l|}{ Proteasome inhibitor } \\
\hline bortezomib & $144(49)$ & $28(18)$ & $116(84)$ & \\
\hline carfilzomib & $2(0)$ & $1(0)$ & $1(0)$ & \\
\hline \multicolumn{5}{|l|}{ Immunomodulatory drug } \\
\hline lenalidomide & $68(23)$ & $68(43)$ & $0(0)$ & \\
\hline thalidomide & $34(11)$ & $32(20)$ & $2(1)$ & \\
\hline Other & $48(16)$ & $29(18)$ & $19(14)$ & \\
\hline
\end{tabular}

VMP: bortezomib-melphalan-prednisone; Rd-R: lenalidomide-dexamethasone followed by lenalidomide maintenance. 
95\% CI: 20.9-28.6) or Rd-R (18.6 months, 95\% CI: 1622.4) $(\mathrm{HR}=0.81, P=0.07)$. At 5 years, $15 \%$ and $18 \%$ of patients were alive and free from progression in the VMP and $\mathrm{Rd}-\mathrm{R}$ groups, respectively. In the subgroup analyses (Figure 1), no clear differences in PFS were noted between the VMP and Rd-R groups according to age (in patients $\leq 75$ years, $\mathrm{HR}=0.80$; in patients $>75$ years, $\mathrm{HR}=0.84$, interaction $P=0.85$ ), Karnofsky PS (score 90-100, $\mathrm{HR}=0.73$; score $70-80, \mathrm{HR}=0.86$, interaction $P=0.43$ ), ISS stage (stage $\mathrm{I}, \mathrm{HR}=0.73$; stage II/III, $\mathrm{HR}=0.85$, interaction $P=0.55$ ) and the presence of extramedullary disease (yes, $H R=0.75$; no, $\mathrm{HR}=0.82$, interaction $P=0.78)$. As far as concerns FISHdetermined cytogenetic risk, no difference in PFS was observed among standard-risk patients between the VMP and $\mathrm{Rd}-\mathrm{R}$ groups ( $\mathrm{HR}=0.96,95 \% \mathrm{CI}$ : 0.73-1.28), whereas in high-risk patients, a significant benefit was observed with VMP in comparison with $\mathrm{Rd}-\mathrm{R}(\mathrm{HR}=0.54$, 95\% CI: 0.34-0.84; interaction $P=0.03$ ). The advantage of VMP over Rd-R in high-risk patients was confirmed in the subgroups of single high-risk cytogenetic abnormalities, including del(17p) $(\mathrm{HR}=0.59,95 \% \mathrm{CI}: 0.32-1.09), \mathrm{t}(4 ; 14)$ $(\mathrm{HR}=0.50,95 \%$ CI: 0.27-0.93) and $\mathrm{t}(14 ; 16)(\mathrm{HR}=0.35,95 \%$ CI: 0.09-1.42) (Online Supplementary Figure S1).

The median OS in the overall population was 66.4 months (95\% CI: 57.3-79.7); the median OS was not significantly different between patients treated with VMP (71 months; 95\% CI: 58.2-not reached) or Rd-R (62 months, 95\% CI: 48.2-83.3) ( $\mathrm{HR}=0.85 ; P=0.28)$, with an equivalent proportion of patients alive at 5 years ( $55 \%$ vs. $51 \%$, respectively). In the subgroup analysis (Figure 2), patients $\leq 75$ years old benefited more from VMP than $\mathrm{Rd}$ $\mathrm{R}(\mathrm{HR}=0.71,95 \% \mathrm{CI}: 0.51-1.00)$, whereas patients $>75$ years old benefited more from $\mathrm{Rd}-\mathrm{R}(\mathrm{HR}=1.29,95 \% \mathrm{CI}$ : 0.79-2.13; interaction $P=0.04)$. Similarly to PFS, no significant difference in $O S$ was noted among standard-risk patients between those treated with VMP or Rd-R (HR=1.08, 95\% CI: 0.74-1.58), but among patients with high-risk cytogenetics, an OS advantage was reported for VMP-treated patients over those treated with Rd-R ( $\mathrm{HR}=0.73,95 \% \mathrm{CI}: 0.42-1.26)$ and those with missing data. The advantage for VMP over Rd-R in high-risk patients was confirmed in the subgroups of single highrisk cytogenetic abnormalities, including del(17p) $(\mathrm{HR}=0.81,95 \% \mathrm{CI}: 0.38-1.71), \mathrm{t}(4 ; 14)(\mathrm{HR}=0.74,95 \% \mathrm{CI}$ $0.35-1.56)$, and $\mathrm{t}(14 ; 16)(\mathrm{HR}=0.73,95 \% \mathrm{CI}: 0.13-4.05)$ (Online Supplementary Figure S2).

Multivariate Cox models with three-way interaction were performed to better evaluate the relationship between treatment regimen (VMP vs. Rd-R), age ( $\leq 75$ vs. $>75$ years) and cytogenetic risk (standard vs. high). This analysis confirmed the absence of difference in PFS between VMP and Rd-R according to age in the standardrisk group, while confirmed the PFS benefit from VMP over $\mathrm{Rd}-\mathrm{R}$ in high-risk patients (interaction $P=0.03$ ). With regards to $O S$, older ( $>75$ years), standard-risk patients seemed to benefit more from $\mathrm{Rd}-\mathrm{R}$ than from VMP $(\mathrm{HR}=1.81)$, while the OS advantage from VMP was confirmed in younger ( $\leq 75$ years), standard-risk patients $(\mathrm{HR}=0.83)$. In high-risk patients, the OS benefit was confirmed irrespective of age ( $\leq 75$ years, $\mathrm{HR}=0.75$; $>75$ years, $\mathrm{HR}=0.65$ ) (Table 3).

To better investigate the comparison between VMP and $\mathrm{Rd}-\mathrm{R}$ and the effect of cytogenetics and age we performed a multiple imputation analysis for missing cytogenetics

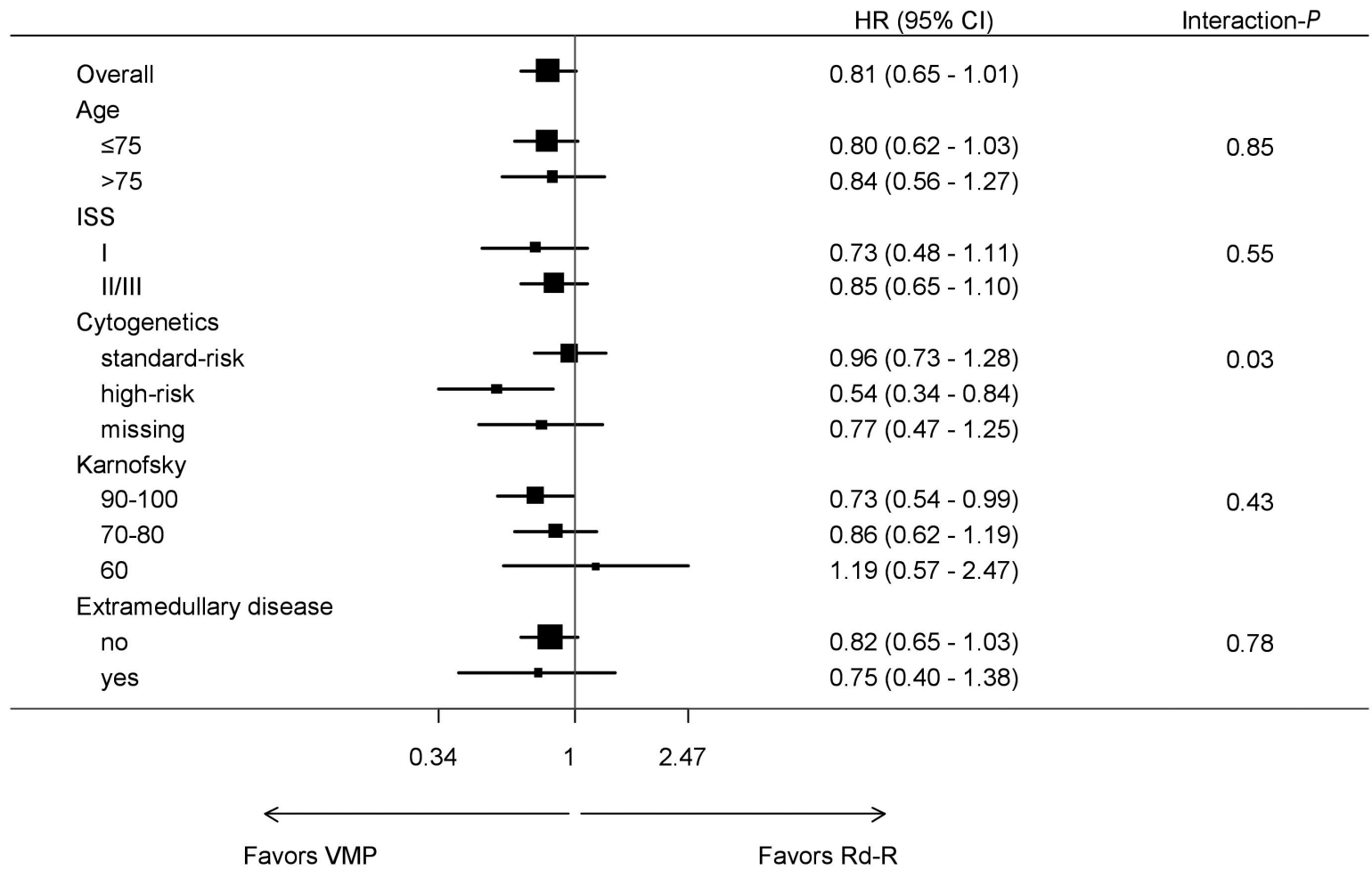

Figure 1. Subgroup analysis of progression-free survival in the intention-to-treat population for patients treated with VMP or Rd-R. VMP: bortezomib-melphalanprednisone; Rd-R: lenalidomide-dexamethasone followed by lenalidomide maintenance; HR: hazard ratio; 95\% Cl: $95 \%$ confidence interval; ISS: International Staging System. 
and ISS stage data. No difference in PFS was observed between the groups treated with VMP or $\mathrm{Rd}-\mathrm{R}(\mathrm{HR}=0.85$, 95\% CI: 0.69-1.04); subgroup analysis confirmed the previous results for cytogenetics, with no difference for standard-risk patients ( $\mathrm{HR}=1.01,95 \% \mathrm{CI}$ : 0.78-1.30); while, in high-risk patients, there was a significant benefit from VMP treatment in comparison with $\mathrm{Rd}-\mathrm{R}$ treatment ( $\mathrm{HR}=0.53$, 95\% CI: 0.34-0.82; interaction $P=0.02$ ) (Online Supplementary Figure S3). No difference in OS was observed between the groups treated with VMP or Rd-R (HR=0.86, 95\% CI: 0.66-1.13); subgroup analysis revealed that VMP was more beneficial than Rd-R for patients $\leq 75$ years ( $\mathrm{HR}=0.72$, 95\% CI: 0.52-0.99), whereas patients $>75$ years benefited more from $\mathrm{Rd}-\mathrm{R}(\mathrm{HR}=1.29,95 \%$ CI: 0.79 2.08; interaction $P=0.05$ ) (Online Supplementary Figure S4).

Multivariate Cox models with the three-way interaction confirmed the results for PFS and OS (Online Supplementary Table S1).

\section{Discussion}

In this pooled analysis of 474 transplant-ineligible patients with newly diagnosed $M M$, we evaluated the impact on survival outcomes of initial treatment, consisting of either a bortezomib-based regimen (VMP) or a lenalidomide-based one ( $\mathrm{Rd}-\mathrm{R}$ ), in different subgroups of patients, focusing on groups with different cytogenetic risk profiles, as determined by FISH. We found no difference between VMP and Rd-R among standard-risk patients, whereas, among high-risk patients, VMP improved PFS $(\mathrm{HR}=0.54)$ and $\mathrm{OS}(\mathrm{HR}=0.73)$ as compared to $\mathrm{Rd}-\mathrm{R}$.

Risk assessment and stratification have long been per- formed in MM, taking into consideration both the aggressiveness of the disease at presentation, based on ISS stage, and its cytogenetic features, determined by either FISH or gene expression profile. Many prognostic factors have been identified in myeloma, the most important ones being chronological and biological age, defined by frailty status in elderly patients, and the presence of chromosomal abnormalities identified by FISH. ${ }^{11}$

Although risk assessment had limited impact on therapeutic choices in the past, with the expanding armamentarium of treatments for MM and the growing evidence of effect modification, it is likely to become a fundamental factor in selecting and tailoring treatment. The International Myeloma Working Group (IMWG) guidelines recommend that all newly diagnosed (ND)MM patients be screened by FISH for chromosomal abnormalities, including del(17p), $\mathrm{t}(4 ; 14)$ and $\mathrm{t}(14 ; 16)$, and that all older patients undergo a geriatric assessment for the evaluation of frailty. ${ }^{19}$ Despite these recommendations, to date no trial has prospectively evaluated the efficacy of standard therapies according to patients' risk status, either based on chromosomal abnormalities identified by FISH or on frailty status. Hence, very limited data are available about the efficacy of current standards of care, such as VMP and $\mathrm{Rd}$, for NDMM patients with high-risk cytogenetics. The VISTA trial did not find any difference between high-risk and standard-risk patients treated with VMP.,5 In the FIRST trial, there was no evidence that lenalidomide improved outcome of patients with high-risk cytogenetics. However, the small number of high-risk patients in both trials makes it difficult to draw definitive conclusions.

In our study, we defined high-risk patients as those carrying at least one cytogenetic abnormality, including
$\mathrm{HR}(95 \% \mathrm{Cl})$

$0.85(0.63-1.14)$

$0.71(0.51-1.00)$

$1.29(0.79-2.13)$

$0.59(0.32-1.08)$

$0.96(0.69-1.34)$

$1.08(0.74-1.58)$

$0.73(0.42-1.26)$

$0.52(0.29-0.95)$

$0.79(0.52-1.20)$

$0.92(0.61-1.38)$

$0.82(0.35-1.91)$

$0.84(0.62-1.15)$

$0.91(0.44-1.89)$
Interaction- $P$

0.04

0.16

0.03

0.86

0.85

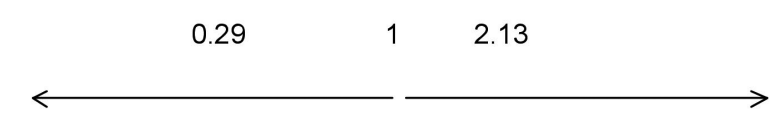

Favors VMP

Figure 2. Subgroup analysis of overall survival in the intention-to-treat population for patients treated with VMP or Rd-R. VMP: bortezomib-melphalan-prednisone; Rd-R: lenalidomide-dexamethasone followed by lenalidomide maintenance; HR: hazard ratio; 95\% CI: 95\% confidence interval; ISS: International Staging System. 
$\operatorname{del}(17 \mathrm{p}), \mathrm{t}(4 ; 14)$ and $\mathrm{t}(14 ; 16)$, consistently with the IMWG recommendations. Major advantages of this study are the number of patients with available cytogenetic data and the fact that all FISH analyses were performed at one centralized facility only.

In our analysis, VMP resulted in a $45 \%$ reduction in the risk of death or progression, as compared to that achieved with $\mathrm{Rd}-\mathrm{R}(\mathrm{HR}=0.54)$, in the high-risk group, with the PFS benefit being confirmed across the single cytogenetic abnormalities, whereas no significant difference in PFS was found in the standard-risk group ( $\mathrm{HR}=0.96$, interaction $P=0.03)$. Furthermore, high-risk patients treated with VMP had a reduced risk of death (HR: 0.73), confirmed across all cytogenetic subgroups, in comparison with patients treated with Rd-R, while no difference was noted between patients treated with VMP or Rd-R within the standardrisk group ( $\mathrm{HR}=1.08)$. However, we could not distinguish the role of melphalan from that of bortezomib in improving the outcome of high-risk patients.

The PFS benefit for VMP over Rd-R in high-risk patients was seen in both younger ( $\leq 75$ years; $H R=0.75$ ) and older patients ( $>75$ years; $H R=0.19)$, while in older, standard-risk patients VMP and Rd-R had similar effects on outcomes $(\mathrm{HR}=0.96)$. Similarly, while the OS benefit among high-risk patients was independent of age (patients $\leq 75$ years, $\mathrm{HR}=0.75$; patients $>75$ years, $\mathrm{HR}=0.65$ ), older patients with standard-risk cytogenetics benefited less from VMP (patients $>75$ years, $\mathrm{HR}=1.81$ ) than they did from $\mathrm{Rd}-\mathrm{R}$.

No data about the patients' frailty at diagnosis were available in the GIMEMA-MM-03-05 trial, and chronological age was the only parameter that could be evaluated in the two trials included in this analysis.

In order to better assess the efficacy of approved upfront regimens according to cytogenetic risk, we restricted our analysis to patients randomized to the VMP arm of the GIMEMA-MM-03-05 trial and the Rd-R arm of the EMN01 trial, since both VMP and Rd are approved combinations for patients with newly diagnosed $M M$ who are ineligible for transplantation. Of note, in the EMN01-trial patients in the $\mathrm{Rd}-\mathrm{R}$ arm received nine cycles of $\mathrm{Rd}$ followed by lenalidomide maintenance or lenalidomide-prednisone maintenance until progression (lenalidomide 10 $\mathrm{mg} /$ day), whereas in the standard approved regimen, $\mathrm{Rd}$ is administered continuously until progression. ${ }^{15}$ This could in part explain the inferior PFS in the EMN01 trial (21 months) as compared to that in the FIRST trial (26

Table 3. Multivariate Cox models with three-way interaction between treatment type, age and cytogenetics, adjusted for International Staging System stage, Karnofsky Performance Status, extramedullary disease and age as a continuous variable.

\begin{tabular}{lcc} 
Main analysis & $\begin{array}{c}\text { PFS } \\
\text { HR* }(9.5 \% \text { CI })\end{array}$ & $\begin{array}{c}\text { OS } \\
\text { HR* }(95 \% \text { CI })\end{array}$ \\
\hline Standard-risk cytogenetics - age $\leq 75$ & $0.92(0.67-1.27)$ & $0.83(0.53-1.29)$ \\
Standard-risk cytogenetics - age $>75$ & $0.96(0.56-1.66)$ & $1.81(0.94-3.49)$ \\
\hline High-risk cytogenetics - age $\leq 75$ & $0.75(0.45-1.26)$ & $0.75(0.39-1.43)$ \\
High-risk cytogenetics - age $>75$ & $0.19(0.07-0.52)$ & $0.65(0.21-2.04)$ \\
\hline 3-way interaction- $P$ & 0.03 & 0.23 \\
2-way cytogenetics interaction- $P$ & 0.03 & 0.23 \\
\hline 2-way age interaction- $P$ & 0.85 & 0.04
\end{tabular}

PFS: progression-free survival; OS: overall survival; HR: hazard ratio; $95 \% \mathrm{CI}$ : $95 \%$ confidence interval. months). ${ }^{7,15}$ On the other hand, we recently presented, at an American Society of Hematology meeting, preliminary results of a randomized phase III study comparing standard $\mathrm{Rd}$ to $\mathrm{Rd}-\mathrm{R}$ in older, intermediate fit patients, defined by the IMWG frailty score, ${ }^{19}$ showing no difference between continuous $\mathrm{Rd}$ and $\mathrm{Rd}-\mathrm{R}$ in terms of PFS $(\mathrm{HR}=0.93$, 95\% CI: 0.64-1.34; $P=0.681)$ and $\mathrm{OS}(\mathrm{HR}=0.73$, 95\% CI: $0.40-1.33 ; P=0.306) .{ }^{20}$

In the VISTA trial, bortezomib was given twice weekly during cycles 1 to 4 and once weekly thereafter, while in the GIMEMA-MM-03-05-trial half of the patients in the VMP arm $(n=191,51 \%)$ received once-weekly bortezomib. However, we had previously shown that the once weekly schedule was equally effective as the twice weekly one in a subgroup analysis of the GIMEMA-MM-03-05 study, potentially due to a more tolerable safety profile of once weekly bortezomib. ${ }^{13,14}$ This might explain a somewhat more favorable median PFS with VMP in the GIMEMA-MM-03-05 study (median, 24.8 months) as compared to the PFS reported in the VISTA trial (median, 21.7 months $){ }^{4}$

The major limitation of this unplanned cross-trial comparison is the absence of randomization between the two treatments, so the results should be interpreted with particular caution. Despite similar eligibility criteria and a comparable follow-up of more than 5 years, there were some significant differences between the two populations. In fact, patients treated with Rd-R were significantly older and patients treated with VMP had a significantly higher creatinine level. Another limitation is that patients enrolled in the two source trials had to meet strict inclusion and exclusion criteria.

Despite these caveats, a head-to-head comparison between VMP and Rd is currently lacking, as is a prospective evaluation of the different treatments in high-risk and standard-risk MM patients. To the best of our knowledge, this is the first study that has pooled together and analyzed a large series of transplant-ineligible patients with NDMM treated with either a bortezomib- or lenalidomide-based combination, with the aim of providing an answer to the burning question of the optimal upfront treatment for NDMM patients according to their cytogenetic risk.

Our results suggest that the doublet regimen $\mathrm{Rd}$ may be a suboptimal option for patients with high-risk cytogenetics, further supporting the 2016 IMWG recommendations that a triplet regimen containing an IMiD and a PI should be used in this setting. ${ }^{9}$ In this light, a major step forward has been made with the results of the Southwest Oncology Group (SWOG) S0777, which showed superior response rate, PFS and OS with the triplet regimen bortezomib-lenalidomide-dexamethasone (VRD) than with $\mathrm{Rd}$ in NDMM without intention to immediate transplantation. ${ }^{21}$ Of note, the longest PFS in high-risk patients was obtained with VRD (38 months). Nevertheless, the analysis was based on only 44 high-risk patients.

The selection of treatment in elderly patients should also consider the risk of toxicity and the capability to tolerate treatment, since advanced age and the occurrence of severe adverse events may negatively affect survival. ${ }^{22}$ In our analysis, this was particularly evident in standard-risk patients, in whom no difference was found between VMP and $\mathrm{Rd}-\mathrm{R}$ and the benefit of $\mathrm{Rd}-\mathrm{R}$ was more evident in patients over 75 years. In this context, the presence of specific comorbidities (such as peripheral neuropathy or renal insufficiency), older age (>75 years) or the presence of 
frailty, as well as the patient's compliance and treatment preference should be considered when choosing treatment.

Our results highlight the importance of performing FISH analysis in all cases of NDMM for risk stratification. Treatment decisions in elderly patients ineligible for transplant are extremely complex, since they should take into consideration not only the biology and the stage of the disease, but also the characteristics of patients (frailty status, comorbidities, hospitalization, concomitant medications, social support, compliance) and goals of care (depth of response or disease control). Therefore, both VMP and Rd are valid options for transplant-ineligible NDMM. Nevertheless, VMP could be preferred in patients with high-risk cytogenetics and severe renal insufficiency, whereas continuous $\mathrm{Rd}$ could be the treatment of choice in standard-risk patients, particularly those over 75 years, or if oral administration and avoidance of peripheral neuropathy are major considerations.

The results of this analysis are based on a selected population, including patients enrolled in clinical trials. Nevertheless, an ongoing trial will prospectively compare these two standard treatments, VMP and continuous Rd, and the impact of cytogenetics on outcomes in an unselected population of patients $\geq 65$ years with $\mathrm{MM}$ in every day clinical practice (Real MM trial, ClinicalTrials.gov Identifier: NCT03829371).
Better treatment options and newer combinations are needed for high-risk disease. Recent trials incorporating the first-in-class monoclonal antibody anti-CD38 daratumum$\mathrm{ab}$ combined with VMP (Dara-VMP) ${ }^{23}$ or Rd (Dara-Rd) ${ }^{24}$ showed that these regimes significantly reduced the risk of progression or death by $50 \%$ and $44 \%$, respectively, as compared to standard VMP (Dara-VMP vs. VMP: median PFS not reached vs. 18.1 months, $\mathrm{HR}=0.50$, 95\% CI: 0.380.65 ) and Rd (Dara_Rd vs. Rd: median PFS not reached vs. 31.9 months, $\mathrm{HR}=0.56,95 \% \mathrm{CI}: 0.43-0.73)$. The benefit of Dara-VMP and Dara-Rd was evident in most of the subgroups analyzed; however the addition of daratumumab did not seem not to overcome the poor prognosis of highrisk patients.

Ongoing trials testing multi-drug combinations including IMiD, second-generation PI, such as carfilzomib and ixazomib, and monoclonal antibodies in the frontline setting will evaluate and, potentially, improve the outcome of high-risk patients.

\section{Acknowledgments}

The authors are grateful to the investigators of all participating sites and the patients who took part in the source studies. The authors also thank the data managers Debora Caldarazzo and Angela Jiang, and the nurses Rosalia Capobianco and Giacomo Castorina from the Torino site.

\section{References}

1. Palumbo A, Anderson K. Multiple myeloma. N Engl J Med 2011;364(11):1046-1060.

2. Howlader N, Noone A, Krapcho M, et al. SEER Cancer Statistics Review, 1975-2014. Bethesda, US-MD

3. Moreau P, San Miguel J, Sonneveld P, et al. Multiple myeloma: ESMO clinical practice guidelines for diagnosis, treatment and follow-up. Ann Oncol Off J Eur Soc Med Oncol. 2017;28(Suppl_4):iv52-iv61.

4. San-Miguel JF, Schlag R, Khuageva NK, et al. Bortezomib plus melphalan and prednisone for initial treatment of multiple myeloma. $\mathrm{N}$ Engl J Med. 2008;359(9):906-917.

5. San-Miguel JF, Schlag R, Khuageva NK, et al. Persistent overall survival benefit and no increased risk of second malignancies with bortezomib-melphalan-prednisone versus melphalan-prednisone in patients with previously untreated multiple myeloma. J Clin Oncol. 2013;31(4):448-455.

6. Benboubker L, Dimopoulos MA, Dispenzieri A, et al. Lenalidomide and dexamethasone in transplant-ineligible patients with myeloma. N Engl J Med. 2014;371(10): 906-917

7. Facon T, Dimopoulos MA, Dispenzieri A, et al. Final analysis of survival outcomes in the phase 3 FIRST trial of up-front treatment for multiple myeloma. Blood. 2018;131(3):301310.

8. Greipp PR, San Miguel J, Durie BGM, et al. International staging system for multiple myeloma. J Clin Oncol. 2005;23(15):34123420.

9. Sonneveld P, Avet-Loiseau H, Lonial S, et al. Treatment of multiple myeloma with highrisk cytogenetics: a consensus of the International Myeloma Working Group. Blood. 2016;127(24):2955-2962.

10. Avet-Loiseau H, Hulin C, Benboubker L, et al. Impact of cytogenetics on outcomes of transplant-ineligible patients with newly diagnosed multiple myeloma treated with continuous lenalidomide plus low-dose dexamethasone in the first (MM-020) Trial. Blood. 2015;126(23): 730.

11. Larocca A, Dold SM, Zweegman S, et al Patient-centered practice in elderly myeloma patients: an overview and consensus from the European Myeloma Network (EMN). Leukemia. 2018;32(8):1697-1712.

12. Palumbo A, Bringhen S, Rossi D, et al. Bortezomib-melphalan-prednisone-thalidomide followed by maintenance with bortezomib-thalidomide compared with bortezomib-melphalan-prednisone for initial treatment of multiple myeloma: a randomized controlled trial. J Clin Oncol. 2010;28 (34):5101-5109.

13. Palumbo A, Bringhen S, Larocca A, et al. Bortezomib-melphalan-prednisone-thalidomide followed by maintenance with bortezomib-thalidomide compared with bortezomib-melphalan-prednisone for initial treatment of multiple myeloma: updated follow-up and improved survival. J Clin Oncol. 2014;32(7):634-640.

14. Bringhen S, Larocca A, Rossi D, et al. Efficacy and safety of once-weekly bortezomib in multiple myeloma patients. Blood. 2010;116(23):4745-4753.

15. Magarotto V, Bringhen S, Offidani M, et al. Triplet vs doublet lenalidomide-containing regimens for the treatment of elderly patients with newly diagnosed multiple myeloma. Blood. 2016;127(9):1102-1108.

16. Carpenter JR, Kenward MG. Multiple Imputation and its Application. Chichester, UK: John Wiley \& Sons, Ltd. 2015.

17. Bartlett JW, Seaman SR, White IR, Carpenter JR. Multiple imputation of covariates by fully conditional specification: accommodating the substantive model. Stat Methods Med Res. 2015;24(4):462-487.
18. Quartagno M, Carpenter JR. Multilevel multiple imputation in presence of interactions, non-linearities and random slopes. 49th Scientific Meeting of the Italian Statistical Society.

19. Palumbo A, Bringhen S, Mateos M-V, et al. Geriatric assessment predicts survival and toxicities in elderly myeloma patients: an International Myeloma Working Group report. Blood. 2015;125(13):2068-2074.

20. Larocca A, Salvini M, De Paoli L, et al Efficacy and feasibility of dose/scheduleadjusted Rd-R vs. continuous $\mathrm{Rd}$ in elderly and intermediate-fit newly diagnosed multiple myeloma (NDMM) patients: RV-MM-PI0752 phase III randomized study. 2018;132(Suppl 1):305.

21. Durie BGM, Hoering A, Abidi $M H$, et al. Bortezomib with lenalidomide and dexamethasone versus lenalidomide and dexamethasone alone in patients with newly diagnosed myeloma without intent for immediate autologous stem-cell transplant (SWOG S0777): a randomised, open-label, phase 3 trial. Lancet. 2017;389(10068):519527.

22. Bringhen S, Mateos MV, Zweegman S, et al. Age and organ damage correlate with poor survival in myeloma patients: meta-analysis of 1435 individual patient data from 4 randomized trials. Haematologica. 2013;98(6): 980-987.

23. Mateos M-V, Dimopoulos MA, Cavo M, et al. Daratumumab plus bortezomib, melphalan, and prednisone for untreated myeloma. N Engl J Med 2018;378(6):518-528.

24. Facon T, Kumar SK, Plesner T, et al. Phase 3 randomized study of daratumumab plus lenalidomide and dexamethasone (D-Rd) versus lenalidomide and dexamethasone $(\mathrm{Rd})$ in patients with newly diagnosed multiple myeloma (NDMM) ineligible for transplant (MAIA). Blood. 2018;132(Suppl 1):LBA-2 